\title{
Chronic treatment with ginsenoside Rg3 induces Akt- dependent senescence in human glioma cells
}

\author{
SEONG SIN ${ }^{1,2^{*}}$, SUNG YOUNG KIM ${ }^{2 *}$ and SUNG SU KIM ${ }^{1,2}$ \\ ${ }^{1}$ Department of Oriental Medicine, Kyung Hee University College of Oriental Medicine, Seoul 130-701; \\ ${ }^{2}$ Soram Cancer and Immunotherapy Research Center, Seoul 135-090, Republic of Korea
}

Received April 6, 2012; Accepted June 14, 2012

DOI: 10.3892/ijo.2012.1604

\begin{abstract}
Therapy-induced senescence, an irreversible growth arrest, in cancer cells is regarded as a novel functional target that may improve cancer therapy. 20(S)-ginsenoside Rg3 [20(S)-Rg3], a chemical component extracted from Panax ginseng, has recently emerged as an effective anticancer medicine with evident antitumor effects and no observed toxic adverse reactions. We report here that chronic treatment with $20(\mathrm{~S})-\operatorname{Rg} 3$ in a sub-lethal concentration induced senescence-like growth arrest in human glioma cells. Glioma cells treated with 20(S)-Rg3 showed high expression of senescence-associated $\beta$-galactosidase, followed by upregulation of the CDK inhibitors p21 and p16. Moreover, reactive oxygen species (ROS) generation markedly increased in 20 (S)-Rg3treated cells compared with control cells. Consistently, co-incubation with the antioxidant $\mathrm{N}$-acetyl cysteine interfered with 20(S)-Rg3-induced senescence in glioma cells. In addition, 20(S)-Rg3-induced-activation of Akt was associated with increased ROS levels, and depletion of Akt partially prevented 20(S)-Rg3-induced ROS generation and senescence induction in glioma cells. Furthermore, 20(S)-Rg3-induced senescence was partially rescued when the p53/p21 pathway was inactivated. Our data indicate that 20 (S)-Rg3 induces senescence-like growth arrest in human glioma cancer through the Akt and p53/p21-dependent signaling pathways. This is the first report of a pro-senescent effect of $20(\mathrm{~S})-\mathrm{Rg} 3$ in cancer cells.
\end{abstract}

\section{Introduction}

It is widely accepted that normal somatic cells have intrinsically limited division capacity and reach a non-proliferative

Correspondence to: Professor Sung Su Kim, Soram Cancer and Immunotherapy Research Center, Samsungdong, Kangnamgu, Seoul, Republic of Korea

E-mail: ssukim12@gmail.com

${ }^{*}$ Contributed equally

Key words: ginsenoside Rg3, senescence, glioma, Akt, ROS state called cellular senescence. Senescent cells are characterized by irreversible growth arrest, a typical gene-expression profile, hyporesponsiveness to external stimuli, increased acidic $\beta$-galactosidase activity, and a flat and enlarged morphology. Most tumors contain cell populations that have escaped the senescence barriers to proliferative potential. This capability, known as immortality, could trigger genomic instability and tumorigenesis. Therefore, cellular senescence has been proposed as a major tumor suppression mechanism (1), and therapy-induced senescence represents a novel functional target that may improve cancer therapy (2-5).

Gliomas are the most common primary brain tumor in adults, and high-grade gliomas are almost universally fatal. Despite recent advances in diagnosis and therapy, including surgical resection followed by radiation and chemotherapy, the prognosis for patients with malignant glioma remain unsatisfactory due to the high recurrence rate and relative drug resistance of high-grade glioma cells (6). Conventional anticancer drugs are associated with relatively strong toxic side-effects and drug resistance.

20(S)-ginsenoside $\operatorname{Rg} 3$ [20(S)- $\mathrm{Rg} 3]$ is an effective medicinal chemical compound extracted from Panax ginseng with a $\mathrm{C}_{42} \mathrm{H}_{72} \mathrm{O}_{13}$ framework and $784.3 \mathrm{Da}$ molecular weight (7). 20(S)-Rg3 has been shown to be safe, and recent evidence from in vitro experiments and animal models have demonstrated that $20(\mathrm{~S})-\operatorname{Rg} 3$ possesses a variety of anti-mutagenic and cancer-inhibitory properties (8-12). However, the exact molecular mechanism of its antitumor effects remains unclear.

Here, we show that chronic treatment with 20(S)-Rg3 at a sub-apoptotic concentration caused senescence-like growth arrest in U87 glioma cells and that this was partially reliant on 20(S)-Rg3-induced ROS generation and induction of p53/ p21. Moreover, we found that Akt plays a critical role in 20(S)-Rg3-induced ROS generation and senescence in glioma cells.

\section{Materials and methods}

Materials. U87 glioma cell lines were purchased from American Type Culture Collections (Manassas, VA, USA). These cell lines were cultured in Dulbecco's modified Eagle's medium (HyClone, Logan, UT, USA) supplemented with $10 \%$ fetal bovine serum and $25 \mathrm{U} / \mathrm{ml}$ penicillin/streptomycin. Anti-phospho-p53, and phospho-Akt polyclonal antibody were 
purchased from Cell Signaling Inc. (Danvers, MA, USA). Antibodies for $\mathrm{p} 21, \mathrm{p} 27, \beta$-actin were purchased from Santa Cruz Biotechnology (Santa Cruz, CA, USA). Secondary horseradish peroxidase-conjugated anti-rabbit and anti-mouse antibodies were from Zymed Laboratories (San Francisco, CA, USA); chemiluminescent detection systems were from Pierce (Rockford, IL, USA); and Lipodin-Pro protein transfection reagent was from Abbiotec (San Diego, CA, USA). Small interfering RNA duplexes against Akt, p53, and p21 were purchased from Dharmacon Co. Transfection reagent (Oligofectamine ${ }^{\mathrm{TM}}$ 2000) was purchased from Invitrogen (Eugene, OR, USA). N-acetyl-1-cysteine and doxorubicin were obtained from Calbiochem (San Diego, CA, USA), and 2', 7'-dichlorofluorescein diacetate and MitoSOX was purchased from Invitrogen (Carlsbad, CA, USA). 20(S)-ginsenoside Rg3 were obtained from International laboratory (USA). All other biochemical reagents were from Sigma or Invitrogen.

SA $\beta$-galactosidase staining. Cellular senescence of cells was confirmed by a senescence-associated $\beta$-galactosidase activity assay as described by Dimri et al (13). After being grown in a semi-confluent state, senescence-associated $\beta$-galactosidase, $\mathrm{pH}$ 6.0, activity was examined. Cells were washed with phosphate-buffered saline (PBS) and fixed with $2 \%$ paraformaldehyde containing $0.2 \%$ glutaraldehyde in PBS for $5 \mathrm{~min}$ at room temperature (RT). After washing with PBS, cells were incubated with $\beta$-galactosidase reagent $[1 \mathrm{mg} / \mathrm{ml} 5$-bromo-4chloro-3-indolyl- $\beta$-d-galactopyranoside (X-gal), $40 \mathrm{~mm}$ citric acid/sodium phosphate buffer, $\mathrm{pH} 6.0,5 \mathrm{~mm}$ potassium ferrocyanide/potassium ferricyanide, $150 \mathrm{~mm} \mathrm{NaCl}, 2 \mathrm{~mm} \mathrm{MgCl}$ ] at $37^{\circ} \mathrm{C}$.

Western blot analysis. Akt, p16, p21, p27 and p53 band were determined by western blotting. Briefly, cells were lysed with lysis buffer [50 mM Tris pH7.4, $150 \mathrm{mM} \mathrm{NaCl}, 1 \mathrm{mM}$ EDTA pH 8.0, $1 \mathrm{mM}$ protease inhibitor (Roche), $1 \mathrm{mM}$ PMSF, $1 \mathrm{mM}$ $\mathrm{NaF}, 1 \mathrm{mM}$ sodium orthovanadate], and protein contents were determined using Bradford reagent. Equal amounts of protein $(40 \mu \mathrm{g})$ were then separated by SDS-PAGE and transferred to nitrocellulose membranes (Schleicher \& Schuell BioScience Inc). After blocking with TBS containing Tween-20 in the presence of $2.5 \%$ nonfat dry milk, the membranes were incubated with the primary antibodies at $4^{\circ} \mathrm{C}$ for $16 \mathrm{~h}$. Secondary antibodies were added for $1 \mathrm{~h}$ at RT. The antibody-antigen complexes were detected using the ECL detection system (Pierce).

Analysis of apoptosis by Annexin $V$ staining. The amount of phosphatidylserine (PS) on cell surfaces (a measure of apoptosis) was determined using an Annexin V-FITC apoptosis detection kit (Abcam, USA), according to the manufacturer's instructions. Briefly, following $\mathrm{H}_{2} \mathrm{O}_{2}$ or staurosporine treatment, cells were harvested and washed twice with PBS. The cells were then resuspended in $0.5 \mathrm{ml}$ of cold $1 \mathrm{X}$ binding buffer (10 mM Hepes, $\mathrm{pH} 7.4,150 \mathrm{mM} \mathrm{NaCl}, 2.5 \mathrm{mM} \mathrm{CaCl}_{2}$, $1 \mathrm{mM} \mathrm{MgCl}{ }_{2}, 4 \% \mathrm{BSA}$ ), and $2.5 \mu 1$ of Annexin V-FITC (fluorescein isothiocyanate) was added. Following incubation for $15 \mathrm{~min}$, stained cells were analyzed by flow cytometry (Becton-Dickinson FACSorter) using CELLQuest software. Error bars represent standard deviations of the means.
RNA interference and transfection. U87 cells were plated in a $100 \mathrm{~mm}$ dish, transfected with $0.5 \mathrm{nmol}$ of siRNA and Oligofectamine reagent in serum-free medium and incubated for $4 \mathrm{~h}$ at $37^{\circ} \mathrm{C}$ in a $\mathrm{CO}_{2}$ incubator. Following incubation, the cells were supplemented with growth medium containing $10 \%$ fetal bovine seerum. Cells were harvested after $72 \mathrm{~h}$.

Measurement of intracellular ROS level. Cells were stained with $50 \mu \mathrm{M}$ of DCF-DA for $30 \mathrm{~min}$ and then harvested. For quantitation of mitochondrial ROS, the cells were incubated with $0.2 \mu \mathrm{M}$ MitoSOX for $30 \mathrm{~min}$ at $37^{\circ} \mathrm{C}$, washed with PBS, trypsinized, collected in PBS, and analyzed on a FACSCalibur. To examine the effect of $\mathrm{N}$-acetylcysteine (NAC), cells were treated with $20 \mathrm{mM}$ of NAC for 2 days.

3-(4,5-dimethylthiazol-2-yl)-2,5-diphenyltetrazolium (MTT) assays. Cells were plated in 24-well tissue culture plates and allowed to attach overnight. MTT was added to each well to a final concentration of $200 \mu \mathrm{g} / \mathrm{ml}$, and cells were incubated for $4 \mathrm{~h}$. After removing the medium completely, the formazan product was solubilized with dimethylsulfoxide. Optical densities (OD) were measured at $490 \mathrm{~nm}$. Each experiment was performed three times. Error bars represent standard deviations of the means.

Statistical analysis. Data are presented as the means \pm S.D. and p-values were calculated using a Student's t-test. A value of $p<0.05$ was considered to indicate a statistically significant difference. All data presented are the representative of at least three separate experiments.

\section{Results}

Low or high doses of 20(S)-Rg3 induces either senescence or apoptosis, respectively, in glioma cells. To investigate the growth-regulatory activity of $20(\mathrm{~S})-\mathrm{Rg} 3$ in glioma cells, we treated glioma cells with various concentrations of 20(S)-Rg3. Exposure of U87 cells to 20(S)-Rg3 for 3 days resulted in a dose-dependent inhibition of cell proliferation (Fig. 1A and B). Concentrations of $\geq 10 \mu \mathrm{M} 20$ (S)-Rg3 suppress the growth of U87 cells (Fig. 1B) whereas concentrations $<10 \mu \mathrm{M}$ exhibit little effect (data not shown). Next we examined the involvement of apoptosis in 20(S)-Rg3-induced growth arrest. Direct detection of apoptosis with Annexin V staining showed that apoptotic cells were not significantly increased in a sublethal dose of $20(\mathrm{~S})-\operatorname{Rg} 3(10 \mu \mathrm{M}, 20 \mu \mathrm{M})$ compared with a high dose of $20(\mathrm{~S})-\operatorname{Rg} 3(50 \mu \mathrm{M}, 100 \mu \mathrm{M})$ (Fig. 1C). We further examined the effects of chronic exposure of sub-apoptotic 20(S)-Rg3 on cell proliferation. Direct cell count assay indicated that the growth of U87 cells was gradually inhibited by chronic exposure of a sublethal dose of 20(S)-Rg3, which became more obvious on day 9 (Fig. 1D). 20(S)-Rg3 $(20 \mu \mathrm{M})$ completely suppressed cell proliferation for at least nine days, while inducing only modest levels of cell death (data not shown). To test the possibility that cell growth arrest in response to $20(\mathrm{~S})-\mathrm{Rg} 3$ was caused by the induction of cellular senescence, we treated glioma cells with various sublethal concentrations of $20(\mathrm{~S})-\mathrm{Rg} 3$ and examined the expression of senescence-associated- $\beta$-galactosidase (SA- $\beta$-gal), a commonly accepted marker of senescence. As 
A

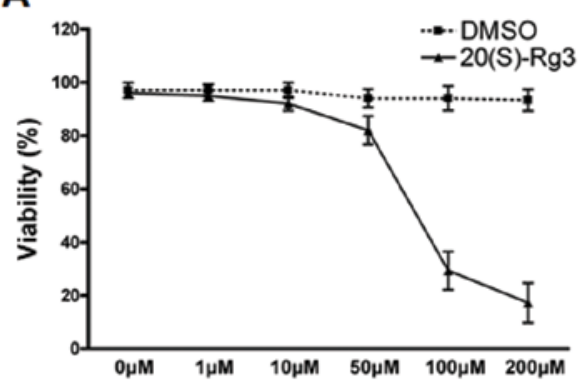

C

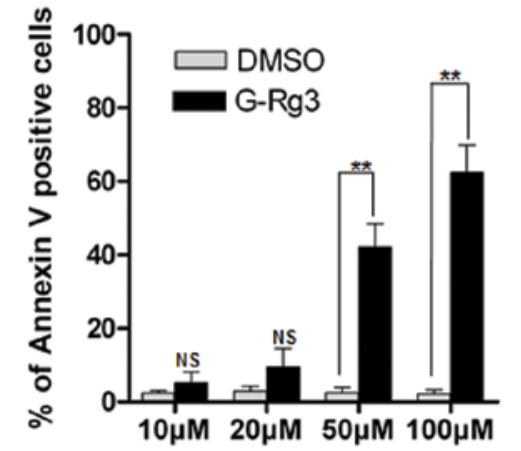

E

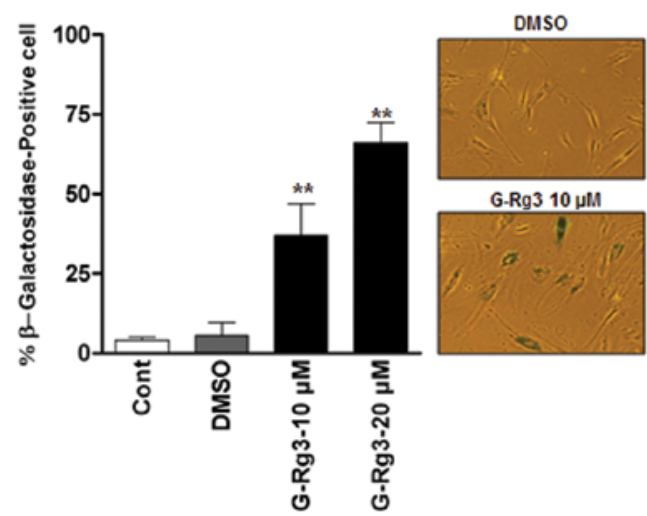

B

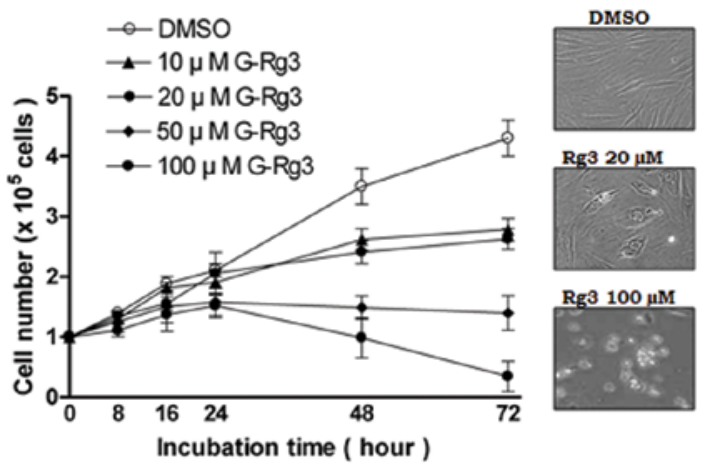

D

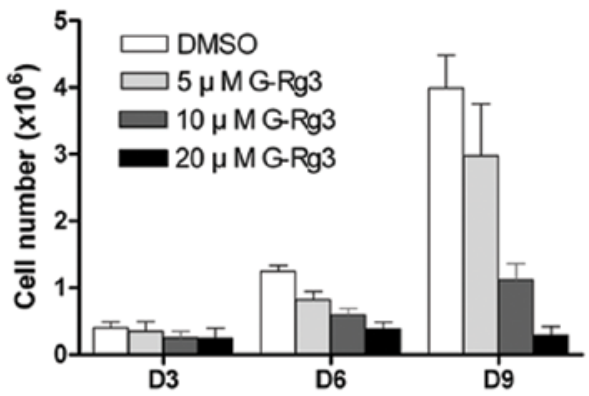

F

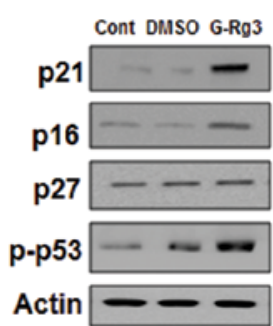

Figure 1. Chronic treatment of 20(S)-Rg3 in U87 cells induces cell senescence. (A) U87 cells were treated for $72 \mathrm{~h}$ with the indicated concentrations of 20 (S)$\mathrm{Rg} 3$ and the relative cell viability was determined by measuring the absorbance or optical density at $490 \mathrm{~nm}$ (OD490) in the MTT staining assay. Error bars represent the SE from three independent experiments. (B) U87 cells were treated with the indicated concentrations of 20(S)-Rg3. Cell numbers were counted at the indicated time points. Data are the mean \pm SEM of three independent experiments. (C) U87 cells were treated with the indicated concentrations of 20(S)-Rg3. Cells were stained with Annexin V-FITC. Annexin V determined by flow cytometry. Error bars represent the SE from three independent experiments. A double asterisk (**) denotes $\mathrm{P}<0.01$ in Student's t-test. (D) U87 cells were treated with the indicated concentrations of 20 (S)-Rg3. Cell numbers were counted at the indicated time points. Data are the mean \pm SEM of three independent experiments. (E) U87 cells were treated with empty control, DMSO, $10 \mu \mathrm{M}$ and $20 \mu \mathrm{M} 20$ (S)-Rg3 for 9 days. Cells were analyzed for the senescence-associated $\beta$-galactosidase activity and stained cells were counted. Data are the mean \pm SEM of three independent experiments. (F) U87 cells were treated with empty control, DMSO, or 20 $\mu$ M 20(S)-Rg3. After 9 days, cells were collected and the expression levels of p21, p16, p27 and p-p53 were analyzed by western blotting. $\beta$-actin was used as a loading control.

shown in Fig. 1E, $67 \%$ of U87 cells after chronic 20(S)-Rg3 treatment at a $20 \mu \mathrm{M}$ concentration were stained positively compared with only $\sim 5 \%$ cells with positive staining in the DMSO control group (Fig. 1E). Cells that were positive for SA- $\beta$-gal showed an enlarged and flattened morphology that was consistent with cellular senescence (Fig. 1E). These findings indicated that $20(\mathrm{~S})-\mathrm{Rg} 3$ could induce senescence in addition to apoptosis. An immunoblot analysis showed that the level of p53 and cyclin-dependent kinase inhibitor p21CIP were markedly increased in 20(S)-Rg3-treated cells (Fig. 1F). However, there was no increase in the expression of $\mathrm{p} 27 \mathrm{Kip} 1$ and only a moderate elevation of p16INK4 in 20(S)-Rg3treated U87 cells (Fig. 1F).
20(S)-Rg3 triggers senescence by an increase of mitochondrial ROS level. As reactive oxygen species (ROS) are well-known inducers of senescence, we evaluated whether treatment of glioma cells with 20(S)-Rg3 increases oxidative stress. ROS levels, as assessed by dichlorofluorescein (DCF), were increased after 20(S)-Rg3 treatment of U87 glioma cells (Fig. 2A). As mitochondrial ROS are the major cellular source of ROS, we also examined the fluorescence of MitoSOX Red as a mitochondrial superoxide indicator. Fluorescence intensity of MitoSOX Red was significantly increased in U87 cells, consistent with increased intracellular ROS levels (Fig. 2B). To address the role of ROS in senescence induced by $20(\mathrm{~S})-\operatorname{Rg} 3$, we treated U87 cells with the ROS scavenger N-acetyl-1- 
A

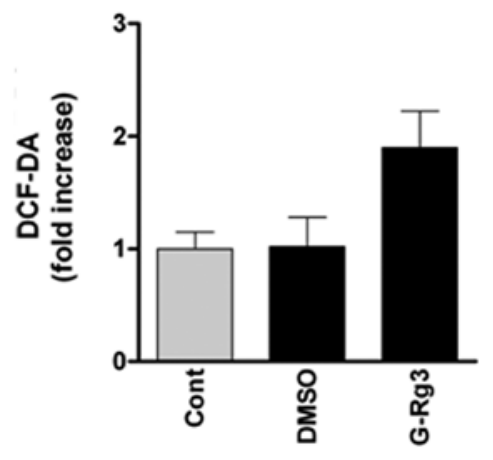

B

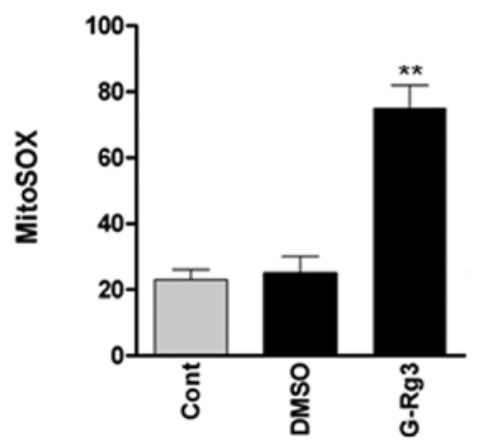

C

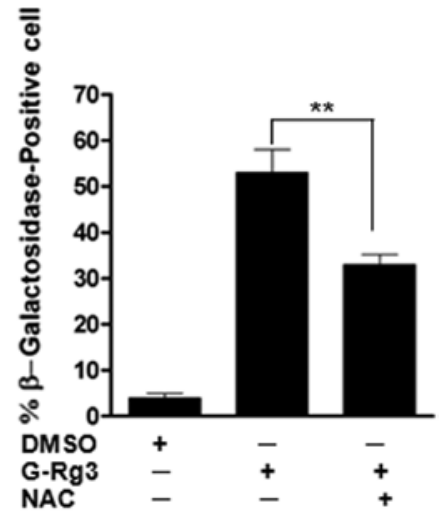

Figure 2. 20(S)-Rg3-induced senescence largely dependent of ROS. (A) U87 cells were treated with empty control, DMSO, and 20 $\mu$ M 20(S)-Rg3 for 9 days. Cells were stained with dichlorofluorescein diacetate (DCF-DA), fixed and immediately analyzed by FACS. (B) U87 cells were treated with empty control, DMSO and $20 \mu \mathrm{M} 20$ (S)-Rg3 for 9 days. Cells were used for flow cytometric analysis of mitochondrial ROS levels using MitoSOX. A double asterisk (**) denotes $\mathrm{P}<0.01$ compared with control cells (ANOVA, Dunnett was used as post-test). (C) U87 cells were treated with DMSO and $20 \mu \mathrm{M} 20$ (S)-Rg3 alone, or pretreated with $20 \mathrm{mM}$ NAC for $16 \mathrm{~h}$. Cells were analyzed for the senescence-associated $\beta$-galactosidase activity and stained cells were counted. A double asterisk $(* *)$ denotes $\mathrm{P}<0.01$ in Student's t-test. The data are the mean \pm SEM of three independent experiments.

cysteine before 20(S)-Rg3 exposure. NAC treatment reduced percentages of SA- $\beta$-gal-positive cells (Fig. $2 \mathrm{C}$ ). These data suggest that elevated ROS levels contribute to senescence induction by 20 (S)-Rg3.

Activation of the Akt contributes to senescence induction by $20(S)-R g 3$ and is associated with elevation of ROS. It has been reported that PI3K/Akt signaling cascades are frequently deregulated in glioma and Akt activation is involved in glioma cell senescence $(14,15)$. To examine whether Akt activity is involved in 20(S)-Rg3-induced senescent glioma cells, we measured phospho-Akt levels. U87 cells treated with $20 \mu \mathrm{M}$ 20(S)-Rg3 were characterized by increases in phosphoAkt (Fig. 3A). To address whether the increase in levels of phospho-Akt is important for the induction of senescence in 20(S)-Rg3-treated human glioma cells, we employed si-RNA against Akt (Fig. 3B). As shown in Fig. 3C, depletion of Akt significantly decreased the SA- $\beta$-gal activity compared with control siRNA-infected cells. As ROS generation has been shown to be involved in the Akt in glioma cells (16), we next examined whether the increased ROS levels caused by $20(\mathrm{~S})-\mathrm{Rg} 3$ occurred as a result of activation of Akt or was a secondary effect of this drug. Transfection of Akt siRNA reduced ROS generation by approximately 32\% (Fig. 3D). These data strongly suggest that AKT may play an important role in regulating cellular ROS status and senescence in glioma cells.

Role of the p53/p21 pathway in the senescence of human glioma cells treated with 20(S)-Rg3. The p53/p21 signaling pathway is a major senescence-triggering pathway in response to various stresses. As shown in Fig. 1F, the treatment of U87 cells with 20(S)-Rg3 significantly increased the expression levels of p53 and p21. Therefore, we examined whether 20(S)-Rg3-mediated senescence requires signaling of the p53/p21 pathway in U87 cells. We transfected U87 cells with p53 and/or p21 si-RNA and looked for senescent phenotypes. As shown in Fig. 4B, p53 siRNA transfected cells showed a significant decrease in SA- $\beta$-Gal activity. Cells transfected with p21 siRNA or co-transfected p53 siRNA showed similar results with p53 depleted cells (Fig. 4B). These data suggest that the p53/p21 plays an essential role in the maintenance of senescence triggered by 20 (S)-Rg3.

\section{Discussion}

The induction of apoptosis was previously defined as the mechanism of 20(S)-Rg3 action for the growth arrest of various tumor cells $(9,10,12)$. In agreement with these reports, our data showed that a high concentration of $20(\mathrm{~S})-\mathrm{Rg} 3$ could induce apoptosis in glioma cells. Here, we showed that 20(S)-Rg3 also induces cellular senescence after prolonged treatment at sublethal drug concentrations.

Cellular senescence, along with mitotic catastrophe and apoptosis, has been proposed as one of the mechanisms mediating the antitumor effects of chemotherapies (2-5). Tumor cells were considered to have lost the ability to senesce, as cellular senescence would provide an important barrier to tumorigenesis $(17,18)$. Previous data have shown that cancer cell senescence can be induced by treatment with chemotherapeutic drugs, radiation, genetic manipulation, or other agents (19). The induction of senescence by chemotherapeutic drugs was dose-dependent and correlated with the growth arrest observed in various cells (2,20-22). In contrast to cell death, however, senescence leaves tumor cells alive and physiologically active (3). In human tumor cell lines treated in vitro and in vivo with differentiating agents, terminal proliferative arrest with minimal toxicity to normal cells has been observed $(1,23)$. Therefore, understanding the mechanism of premature senescence is crucial for the development of safer and more effective cancer treatment strategies.

Gain-of-function mutations in the PI3K/Akt signaling pathway are frequently found in human glioblastomas (14). In mammalian cells, the activation of Akt has been reported to induce proliferation and survival, thereby promoting tumorigenesis (24-26). However, previous studies demonstrated that the constitutive activation of Akt promotes senescence-like arrest of cell growth through the regulation of intracellular ROS levels $(15,16,27,28)$. Thus, Akt-induced growth arrest may be another antitumorigenic mechanism, similar to 
A
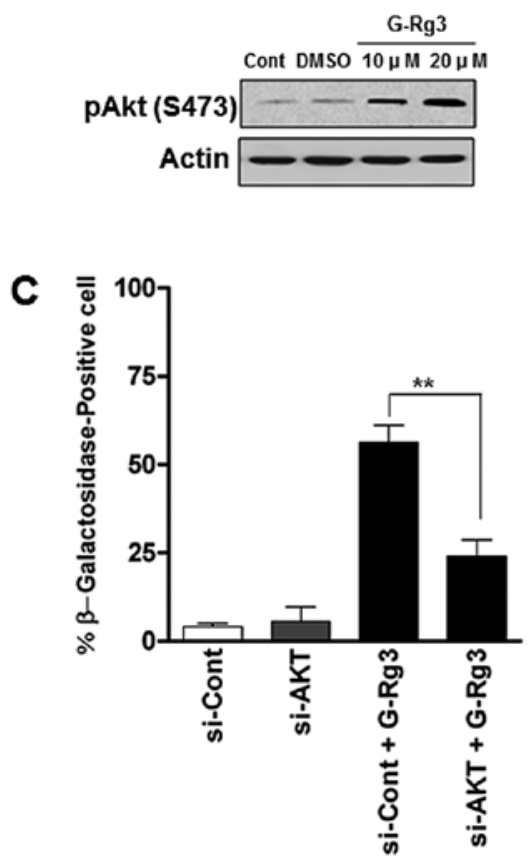

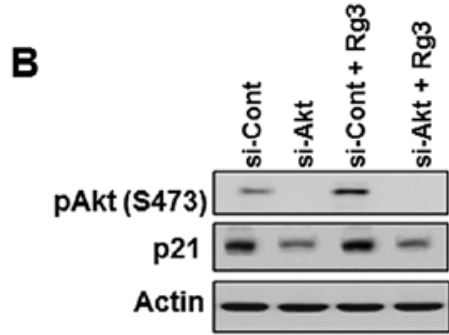

D

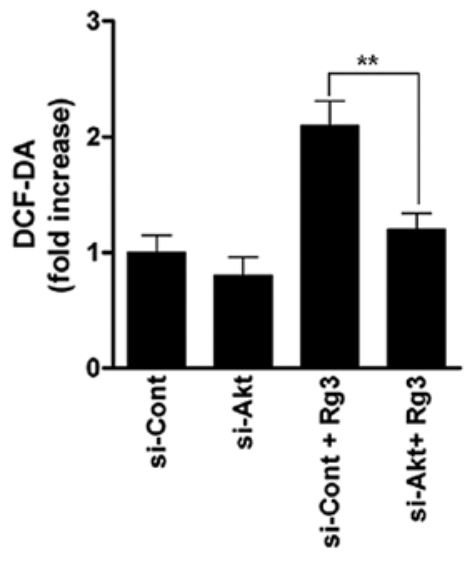

Figure 3. Akt is essential for the induction of 20(S)-Rg3-induced glioma cell senescence. (A) U87 cells were treated with empty control, DMSO, $10 \mu \mathrm{M}$ and $20 \mu \mathrm{M} 20$ (S)-Rg3 for 9 days. Cells were collected and the expression levels of pAkt (S473) were analyzed by western blotting. $\beta$-actin was used as a loading control. (B) U87 cells transfected with $200 \mathrm{nM}$ siRNA for $72 \mathrm{~h}$. U87 cells transfected with $200 \mathrm{nM}$ siRNA every 3 days for 12 days. Three days after the first transfection, cells were treated with $20 \mu \mathrm{M} 20$ (S)-Rg3 for 9 days. Cells were collected and the expression levels of pAkt, p21 were analyzed by western blotting. $\beta$-actin was used as a loading control. (C) U87 cells transfected with $200 \mathrm{nM}$ siRNA every 3 days for 12 days. Three days after the first transfection, cells were treated with $20 \mu \mathrm{M} 20(\mathrm{~S})-\mathrm{Rg} 3$ for 9 days then cells were used for $\beta$-galactosidase analysis and stained cells were counted. (D) U87 cells transfected with $200 \mathrm{nM}$ siRNA against Akt every 3 days for 9 days, followed by the addition of $20 \mu \mathrm{M} 20$ (S)-Rg3 for 9 days. The cells were then stained with dichlorofluorescein diacetate (DCF-DA), fixed and immediately analyzed by FACS. The control scrambled siRNA was labeled as 'Cont'. A double asterisk (**) denotes $\mathrm{P}<0.01$ in Student's t-test. The data are the mean \pm SEM of three independent experiments.

A

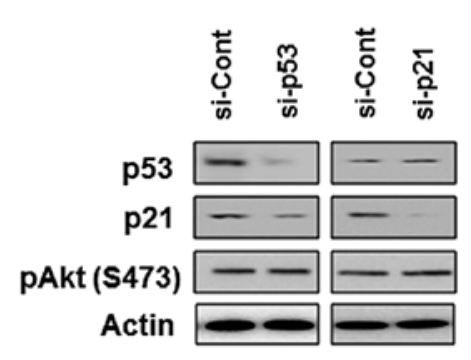

B

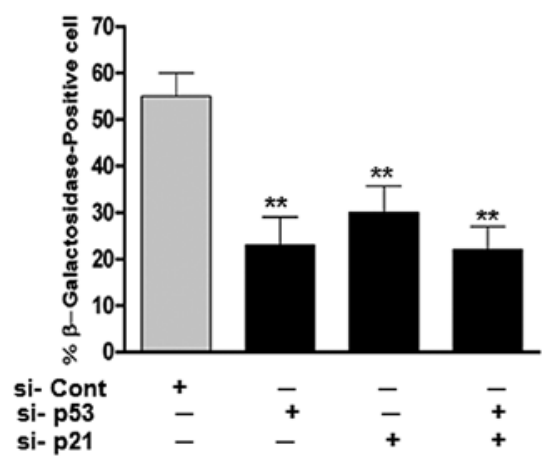

Figure 4. Inactivating the p53/p21 pathway partially protects against 20(S)-Rg3-induced senescence. (A) U87 cells were transfected with si-p53, si-p21 or the control siRNA. After $72 \mathrm{~h}$, cells were collected and the expression levels of p53, p21 and pAkt wre analyzed by western blotting. $\beta$-actin was used as a loading control. (B) U87 cells were transfected with $200 \mathrm{nM}$ siRNA every 3 days for 12 days. Three days after the first transfection, cells were treated with $20 \mu \mathrm{M} 20$ (S)-Rg 3 for 9 days then cells were used for $\beta$-galactosidase analysis and stained cells were counted. The control scrambled siRNA was labeled as 'Cont'. A double asterisk ( $* *$ ) denotes $\mathrm{P}<0.01$ compared with control cells (ANOVA, Dunnett was used as post-test). The data are the mean \pm SEM of three independent experiments.

Ras-induced senescence $(29,30)$. In this study, we revealed that 20(S)-Rg3-induced senescence is associated with Akt activation, and depletion of Akt partially prevented $\mathrm{Rg} 3$-induced ROS generation and senescence induction in glioma cells. Elevated intracellular ROS levels have been widely accepted as triggers of cellular senescence (31-33). Senescent cells increase ROS generation $(34,35)$, and premature cellular senescence can be readily induced by sub-lethal doses of pro-oxidants $(36,37)$. In the present study, ROS levels were elevated during 20(S)-Rg3-induced senescence, and the ROS 
scavenger NAC reverted 20(S)-Rg3-induced senescent phenotypes. However, decreases in intracellular ROS levels by NAC did not affect 20(S)-Rg3-induced Akt activation (data not shown). These data suggest that 20(S)-Rg3 induces senescence by a mechanism largely dependent on Akt/ROS signaling. Furthermore, 20(S)-Rg3-induced arrest was accompanied by substantial increases in p53 and p21, and the depletion of p53 or $\mathrm{p} 21$ prevented $20(\mathrm{~S})-\mathrm{Rg} 3$-induced premature senescence in U87 cells. Thus, senescence may occur in a manner dependent on the p53/p21 pathway. However, further studies are required to determine the exact mechanism involved.

In summary, our study presented a new anticancer mechanism of $20(\mathrm{~S})-\operatorname{Rg} 3$ action by inducing senescence. Chronic treatment with $20(\mathrm{~S})-\operatorname{Rg} 3$ at a sub-apoptotic concentration elicits ROS generation via Akt activation and p53/p21-dependent senescence-like growth arrest in glioma cells. Our study provides useful insight for the future development of 20(S)$\mathrm{Rg} 3$ as a novel class of anticancer agents.

\section{Acknowledgements}

This project was supported by a research grant from the Soram Cancer and Immunotherapy Research Center (Republic of Korea).

\section{References}

1. Vergel M, Marin JJ, Estevez P and Carnero A: Cellular senescence as a target in cancer control. J Aging Res 2011: 725365, 2010.

2. Schmitt CA: Senescence, apoptosis and therapy - cutting the lifelines of cancer. Nat Rev Cancer 3: 286-295, 2003.

3. Shay JW and Roninson IB: Hallmarks of senescence in carcinogenesis and cancer therapy. Oncogene 23: 2919-2933, 2004.

4. Hornsby PJ: Senescence as an anticancer mechanism. J Clin Oncol 25: 1852-1857, 2007.

5. Ventura A, Kirsch DG, McLaughlin ME, et al: Restoration of p53 function leads to tumour regression in vivo. Nature 445: 661-665, 2007.

6. Ohgaki $\mathrm{H}$ and Kleihues P: Genetic pathways to primary and secondary glioblastoma. Am J Pathol 170: 1445-1453, 2007.

7. Kitagawa I, Yoshikawa M, Yoshihara M, Hayashi T and Taniyama T: Chemical studies of crude drugs (1). Constituents of Ginseng radix rubra. Yakugaku Zasshi 103: 612-622, 1983 (In Japanese).

8. Helms S: Cancer prevention and therapeutics: Panax ginseng. Altern Med Rev 9: 259-274, 2004.

9. Yuan HD, Quan HY, Zhang Y, Kim SH and Chung SH: 20(S)-Ginsenoside Rg3-induced apoptosis in HT-29 colon cancer cells is associated with AMPK signaling pathway. Mol Med Rep 3: 825-831, 2010.

10. Wang BS, Zhang LS, Song DM, Zhang JH and Liu YM: Effect of gensenoside Rg3 on apoptosis of Hep-2 and expression of HIF-1alha in human laryngeal cancer cell line under anoxic conditions. Zhong Yao Cai 32: 102-106, 2009 (In Chinese).

11. Kim HS, Lee EH, Ko SR, Choi KJ, Park JH and Im DS: Effects of ginsenosides $\mathrm{Rg} 3$ and $\mathrm{Rh} 2$ on the proliferation of prostate cancer cells. Arch Pharm Res 27: 429-435, 2004.

12. Jian J, Hu ZF and Huang Y: Effect of ginsenoside Rg3 on Pim-3 and Bad proteins in human pancreatic cancer cell line PANC-1. Ai Zheng 28: 461-465, 2009 (In Chinese).

13. Dimri GP, Lee X, Basile G, et al: A biomarker that identifies senescent human cells in culture and in aging skin in vivo. Proc Natl Acad Sci USA 92: 9363-9367, 1995.
14. Solomon DA, Kim JS, Jenkins S, et al: Identification of p18 INK4c as a tumor suppressor gene in glioblastoma multiforme. Cancer Res 68: 2564-2569, 2008.

15. Nogueira V, Park Y, Chen CC, et al: Akt determines replicative senescence and oxidative or oncogenic premature senescence and sensitizes cells to oxidative apoptosis. Cancer Cell 14: 458-470, 2008.

16. Dolado I and Nebreda AR: AKT and oxidative stress team up to kill cancer cells. Cancer Cell 14: 427-429, 2008.

17. Campisi J and d'Adda di Fagagna F: Cellular senescence: when bad things happen to good cells. Nat Rev Mol Cell Biol 8: 729-740, 2007.

18. Schmitt CA: Cellular senescence and cancer treatment. Biochim Biophys Acta 1775: 5-20, 2007.

19. Roninson IB: Tumor cell senescence in cancer treatment. Cancer Res 63: 2705-2715, 2003.

20. te Poele RH, Okorokov AL, Jardine L, Cummings J and Joel SP: DNA damage is able to induce senescence in tumor cells in vitro and in vivo. Cancer Res 62: 1876-1883, 2002.

21. Nardella C, Clohessy JG, Alimonti A and Pandolfi PP: Pro-senescence therapy for cancer treatment. Nat Rev Cancer 11: 503-511, 2011.

22. Lleonart ME, Artero-Castro A and Kondoh H: Senescence induction; a possible cancer therapy. Mol Cancer 8: 3, 2009.

23. Chang BD, Xuan Y, Broude EV, et al: Role of $\mathrm{p} 53$ and p21waf1/ cipl in senescence-like terminal proliferation arrest induced in human tumor cells by chemotherapeutic drugs. Oncogene 18: 4808-4818, 1999.

24. Datta SR, Brunet A and Greenberg ME: Cellular survival: a play in three Akts. Genes Dev 13: 2905-2927, 1999.

25. Blume-Jensen $P$ and Hunter T: Oncogenic kinase signalling. Nature 411: 355-365, 2001.

26. Testa JR and Bellacosa A: AKT plays a central role in tumorigenesis. Proc Natl Acad Sci USA 98: 10983-10985, 2001.

27. Miyauchi H, Minamino T, Tateno K, Kunieda T, Toko H and Komuro I: Akt negatively regulates the in vitro lifespan of human endothelial cells via a p53/p21-dependent pathway. EMBO J 23: 212-220, 2004

28. Lee JJ, Kim BC, Park MJ, et al: PTEN status switches cell fate between premature senescence and apoptosis in glioma exposed to ionizing radiation. Cell Death Differ 18: 666-677, 2011.

29. Serrano M, Lin AW, McCurrach ME, Beach D and Lowe SW: Oncogenic ras provokes premature cell senescence associated with accumulation of p53 and p16INK4a. Cell 88: 593-602, 1997.

30. Wright WE and Shay JW: Cellular senescence as a tumorprotection mechanism: the essential role of counting. Curr Opin Genet Dev 11: 98-103, 2001.

31. Passos JF and Von Zglinicki T: Oxygen free radicals in cell senescence: are they signal transducers? Free Radic Res 40: 1277-1283, 2006.

32. Beckman KB and Ames BN: The free radical theory of aging matures. Physiol Rev 78: 547-581, 1998.

33. Ben-Porath I and Weinberg RA: The signals and pathways activating cellular senescence. Int J Biochem Cell Biol 37: 961-976, 2005.

34. Hagen TM, Yowe DL, Bartholomew JC, et al: Mitochondrial decay in hepatocytes from old rats: membrane potential declines, heterogeneity and oxidants increase. Proc Natl Acad Sci USA 94: 3064-3069, 1997.

35. Lee AC, Fenster BE, Ito $\mathrm{H}$, et al: Ras proteins induce senescence by altering the intracellular levels of reactive oxygen species. J Biol Chem 274: 7936-7940, 1999.

36. Chen Q and Ames BN: Senescence-like growth arrest induced by hydrogen peroxide in human diploid fibroblast F65 cells. Proc Natl Acad Sci USA 91: 4130-4134, 1994.

37. Chen QM, Bartholomew JC, Campisi J, Acosta M, Reagan JD and Ames BN: Molecular analysis of $\mathrm{H} 2 \mathrm{O} 2$-induced senescentlike growth arrest in normal human fibroblasts: p53 and $\mathrm{Rb}$ control G1 arrest but not cell replication. Biochem J 332: 43-50, 1998. 